\title{
Elements of Metrodynamics: the General Setting
}

\author{
Irene Sabadini · Frank Sommen
}

Received: 18 February 2009 / Accepted: 18 May 2009 / Published online: 16 June 2009

(C) Birkhäuser Verlag Basel/Switzerland 2009

\begin{abstract}
In this paper we generalize the study started in (Brackx et al., Oper Theory Adv Appl, Birkhäuser, Basel (2006)) by considering the general case of a nonsingular metric, not necessarily symmetric. We show how this general case allows to derive several interesting cases: besides the symmetric case (see also Brackx et al., 2006) we study the symplectic case and several other systems.
\end{abstract}

Mathematical Subject Classification 30G35

\section{Introduction}

Clifford analysis is a very well established theory which constitutes a powerful generalization of complex analysis. The main object of study are the so-called monogenic functions, which are the nullsolutions of the Dirac operator $\sum_{i} e_{i} \partial_{x_{i}}$. It is also a refinement of harmonic analysis, indeed the Dirac operator factors the $m$-dimensional Laplace operator. In the literature there are several generalizations of the classical Clifford analysis as presented in the classical book [1]: some of them will be quickly revised in Sect. 3. In this paper, we are interested in further extending the ideas of [5] in which the authors started the study of a metric dependent Clifford analysis. In [5] the metric used is given by a tensor of rank 2 which is real, symmetric and definite

Communicated by Daniel Alpay.

I. Sabadini $(\varangle)$

Dipartimento di Matematica, Politecnico di Milano, Via Bonardi, 9, 20133 Milano, Italy

e-mail: irene.sabadini@polimi.it

F. Sommen

Clifford Research Group, Faculty of Sciences, Ghent University, Galglaan 2,

9000 Gent, Belgium

e-mail: Frank.Sommen@UGent.be 
positive, while in this paper we will consider the more general case in which the metric is given by a nondegenerate, real, rank 2 tensor. It is worth noticing that the idea to consider a local metric dependence has already been exploited for Clifford analysis on manifolds and goes back to [12]. However, the novelty of [5] and of this paper is that we consider a global metric tensor whereby the components are interpreted as variables. After revising some basic notions on real and complex Clifford algebras in Sect. 2, in Sect. 3 we introduce the metric dependent Clifford analysis by recalling some basic facts on the symmetric case treated in [5]. Section 4 contains the study of the general case, while in Sect. 5 we give more details on the general case and we focus the attention on the symplectic case. In Sect. 6 we study the covariant standard Fischer duality versus the Hermitian Fischer duality and, finally, in Sect. 7 we introduce the basics on matrix derivatives. For background information on Clifford algebras and Clifford analysis in the orthogonal and symplectic setting, we refer the reader to $[8,9,11,13,15,18]$.

\section{Preliminary Notions}

A Clifford algebra $\mathbb{R}_{m}$ over the field $\mathbb{R}$ of real numbers is defined as the algebra generated by the elements $\left\{e_{1}, \ldots, e_{m}\right\}$ obeying the defining relations $e_{i} e_{j}+e_{j} e_{i}=-2 \delta_{i j}$. An element $a \in \mathbb{R}_{m}$ is of the form $a=\sum_{A \in \wp_{m}} a_{A} e_{A}$ where $\wp_{m}$ is the power set of the set $\{1, \ldots, m\}$ so $A$ is a multi-index $A=\left\{\alpha_{1}, \ldots, \alpha_{k}\right\}, \alpha_{1}<\ldots<\alpha_{k}$ and $e_{A}=e_{\alpha_{1}} \ldots e_{\alpha_{k}}$.

Inside the Clifford algebra $\mathbb{R}_{m}$ thought as a linear space over $\mathbb{R}$ there are the special subspaces $\mathbb{R}_{m}^{k}$ corresponding to the subsets of the so called $k$-vectors:

$$
\mathbb{R}_{m}^{k}=\left\{a \in \mathbb{R}_{m} \mid a=\sum_{|A|=k} a_{A} e_{A}\right\}, \quad a=[a]_{0}+[a]_{1}+\cdots+[a]_{m} .
$$

The subspace of 1-vectors may be identified with the Euclidean space $\mathbb{R}^{m}$ as follows:

$$
\underline{x}: \mathbb{R}^{m} \rightarrow \mathbb{R}_{m}^{1} \quad\left(x_{1}, \ldots, x_{m}\right) \mapsto \sum_{j=1}^{m} x_{j} e_{j}
$$

It is well known that on the space of 1-vectors one can define the following operations:

$$
\begin{aligned}
& \underline{x} \wedge \underline{y}=[\underline{x}]_{2}=\frac{1}{2}[\underline{x}, \underline{y}]=\sum_{j<k} e_{j k}\left(x_{j} y_{k}-x_{k} y_{j}\right) \\
& \underline{x} \cdot \underline{y}=[\underline{x} \underline{y}]_{0}=\frac{1}{2}\{\underline{x}, \underline{y}\}=-\sum_{j=1}^{m} x_{j} y_{j},
\end{aligned}
$$

moreover

$$
\underline{x} \underline{y}=\underline{x} \cdot \underline{y}+\underline{x} \wedge \underline{y} .
$$


We can further generalize the idea of Clifford algebra and consider the Clifford algebra $\mathbb{R}_{p, q}$ which is generated over the field $\mathbb{R}$ by the elements

$$
\varepsilon_{1}, \ldots, \varepsilon_{p} ; e_{1}, \ldots, e_{q}
$$

such that $\varepsilon_{i}^{2}=+1, e_{j}^{2}=-1$ and the anti-commutation relations among the units.

In this paper we will generalize the notion of Clifford algebra by introducing a metric dependence. The idea is not new since it has been introduced in [5] for metrics $g$ which are symmetric and positive definite. Our novelty is that we will consider the most general case of nonsymmetric metrics $g=\left[g_{j k}\right]$. We introduce the following:

Definition 2.1 The Clifford algebra $\mathbb{R}_{m}[g]$ is the algebra over $\mathbb{R}$ generated by the units $e_{1}, \ldots, e_{m}$ satisfying the defining relations

$$
e_{j} e_{k}+e_{k} e_{j}=-2 g_{j k}
$$

Similarly, one can define $\mathbb{C}_{m}[g]$ as the algebra over $\mathbb{C}$ generated by the units $e_{1}, \ldots, e_{m}$.

The subspace of vectors in $\mathbb{R}^{m}$ (resp. $\mathbb{C}^{m}$ ) embeds into $\mathbb{R}_{m}[g]$ (resp. $\mathbb{C}_{m}[g]$ ) through the map (1). Moreover, $\mathbb{R}^{m}$ can be endowed by a metric by defining

$$
\left\langle e_{j}, e_{k}\right\rangle=g_{j k} \quad \text { and } \quad\langle\underline{x}, \underline{y}\rangle=\sum_{j, k} x^{j} y^{k} g_{j k} \text {. }
$$

Note that here we are writing a vector $\underline{x}$ using a contravariant $m$-tuple of coordinates $\left(x^{1}, \ldots, x^{m}\right)$ instead of the covariant notation $\left(x_{1}, \ldots, x_{m}\right)$. We will use one notation or the other according to our needs.

Remark 2.2 The idea of Metrodynamics is to consider the $g_{j k}$ as variables. So the units can also be considered as functions $e_{j}=e_{j}(g)$ depending implicitly on the variables $g_{j k}$ through the defining relations $e_{j} e_{k}+e_{k} e_{j}=-2 g_{j k}$. Note that, in this framework, one might also consider the derivatives $\frac{\partial e_{j}}{\partial g_{k \ell}}$. To obtain the explicit expressions of the elements $e_{j}=e_{j}(g)$ in terms of the components $g_{j k}$ it suffices to construct an over-all embedding Clifford algebra which contains all the algebras $\mathbb{R}_{m}[g]$ as subalgebras i.e. the endomorphism algebra $\operatorname{End}\left(\mathbb{R}_{m}\right)$, which coincides with the Clifford algebra $\mathbb{R}_{m, m}$.

Let us go back to the standard framework of Clifford algebras, and let us consider the algebra of endomorphisms of $\mathbb{R}_{m}$ i.e. $\operatorname{End}\left(\mathbb{R}_{m}\right)$. Its generators $e_{1}, \ldots, e_{m}, e_{1}\left|, \ldots, e_{m}\right|$ are given by

$$
e_{j} \cdot: a \mapsto e_{j} \cdot a ; \quad e_{j} \mid: a \mapsto \tilde{a} e_{j}
$$

where $\sim$ denotes the anti-involution acting as follows:

$$
\tilde{e}_{j}=-e_{j}, \quad \tilde{a b}=\tilde{a} \tilde{b} .
$$


The generators obey the following defining relations:

$$
e_{j} e_{k}\left|=-e_{k}\right| e_{j} ; \quad e_{j}\left|e_{k}\right|=-e_{k}\left|e_{j}\right|, \quad j \neq k, \quad e_{j}^{2}=-1,\left.\quad e_{j}\right|^{2}=+1 .
$$

The following result is well known:

Proposition 2.3 It is possible to identify $\operatorname{End}\left(\mathbb{R}_{m}\right)$ with the ultra-hyperbolic (real) Clifford algebra $\mathbb{R}_{m, m}=A \lg \left\{e_{j}, \varepsilon_{j}, j=1, \ldots, m\right\}$ by the identification $e_{j} \mid \mapsto \varepsilon_{j}$. Note also that by setting $\varepsilon_{j}=i e_{j+m}$ we have

$$
\mathbb{R}_{m, m} \subset \mathbb{C}_{2 m}=\mathbb{C} \otimes \mathbb{R}_{2 m}
$$

In $\mathbb{C}_{2 m}$ we can introduce the elements

$$
\mathfrak{f}_{j}=\frac{1}{2}\left(e_{j}-\varepsilon_{j}\right)=\frac{1}{2}\left(e_{j}-i e_{j+m}\right),
$$

which satisfy the so-called Grassmann relations

$$
\mathfrak{f}_{j}^{2}=0 \quad \mathfrak{f}_{j} \mathfrak{f}_{k}+\mathfrak{f}_{k} \mathfrak{f}_{j}=0 .
$$

Consider now the conjugation which is defined on the elements of the basis as $\bar{e}_{j}=-e_{j}$ and it is such that $\overline{a b}=\bar{b} \bar{a}$. The so-called Hermitian conjugation is defined on $\mathbb{C}_{m}=\mathbb{R}_{m} \oplus i \mathbb{R}_{m}$ as follows

$$
(a+i b)^{\dagger}=\bar{a}-i \bar{b}
$$

If we set

$$
f_{j}^{\dagger}=-\frac{1}{2}\left(e_{j}+\varepsilon_{j}\right)
$$

we have that these elements satisfy

$$
\begin{aligned}
& \mathfrak{f}_{j}^{\dagger}=0 \quad \mathfrak{f}_{j}^{\dagger} \mathfrak{f}_{k}^{\dagger}+\mathfrak{f}_{k}^{\dagger} \mathfrak{f}_{j}^{\dagger}=0 \quad \text { (Grassmann relations), } \\
& \mathfrak{f}_{j} \mathfrak{f}_{k}^{\dagger}+\mathfrak{f}_{k}^{\dagger} \mathfrak{f}_{j}=\delta_{i j} \quad \text { (duality relations). }
\end{aligned}
$$

Definition 2.4 (See $[16,19]$ ) The (complex) algebra $\operatorname{Alg}\left\{\mathfrak{f}_{1}, \ldots, \mathfrak{f}_{m} ; \mathfrak{f}_{1}^{\dagger}, \ldots, \mathfrak{f}_{m}^{\dagger}\right\}$ is $\mathbb{C}_{2 m}$ and the elements $\mathfrak{f}_{j}, \mathfrak{f}_{j}^{\dagger}, j=1, \ldots, m$ form the so-called Witt basis.

The element

$$
I=I_{1} \ldots I_{m}, \quad \text { where } \quad I_{j}=\mathfrak{f}_{j} \mathfrak{f}_{j}^{\dagger} \text {, }
$$

is a primitive idempotent. 
By setting

$$
e_{j}=\mathfrak{f}_{j}-\mathfrak{f}_{j}^{\dagger}, \quad \varepsilon_{j}=-\mathfrak{f}_{j}-\mathfrak{f}_{j}^{\dagger}
$$

we have that $\mathbb{R}_{m}$ can be seen as $\operatorname{Alg}\left\{e_{j}, j=1, \ldots, m\right\}$, while $\mathbb{R}_{m, m}=\operatorname{Alg}\left\{e_{j}, \varepsilon_{j}, j=\right.$ $1, \ldots, m\}$. Moreover we have the conversion relations:

$$
\mathfrak{f}_{j} I=0 \quad e_{j} I=\varepsilon_{j} I=-\mathfrak{f}_{j}^{\dagger} I
$$

For $a \in \mathbb{R}_{m}$ we also have:

$$
e_{j} a I=e_{j}[a] I \quad \varepsilon_{j} a I=\tilde{a} \varepsilon_{j} I=\tilde{a} e_{j} I=e_{j} \mid[a] I .
$$

which provides an alternative way to see the isomorphism $\mathbb{R}_{m, m}=\operatorname{End}\left(\mathbb{R}_{m}\right)$.

\section{Metric Dependent Clifford Analysis}

\subsection{Affine and Hermitian Clifford Analysis}

We start this section by recalling some basics in affine Clifford analysis (see [20]), which is one of the possible generalization of the standard Clifford analysis which deals with functions defined on an Euclidean space $\mathbb{R}^{m}$ with values in $\mathbb{R}_{m}$ that are nullsolutions of the Dirac operator. In this setting, the basic elements are the real variables $x_{j}$, the corresponding partial derivatives $\partial_{x_{j}}$, and the Clifford basis $e_{j}, j=1, \ldots, m$. In affine Clifford analysis, we will work in the setting $\mathbb{R}_{m, m}=\operatorname{End}\left(\mathbb{R}_{m}\right)$, in which it is possible to consider also operators with values in $\operatorname{End}\left(\mathbb{R}_{m}\right)$. We will write the vector variable as $\underline{X}=\sum_{j=1}^{2 m} X_{j} e_{j}$. The $2 m$-tuple $\left(X_{1}, \ldots, X_{m}, X_{m+1}, \ldots, X_{2 m}\right)$ is such that $X_{\ell} \in \mathbb{R}, X_{m+\ell} \in i \mathbb{R}$, for $\ell=1, \ldots, m$. If we set $Y_{j}=i X_{m+j}, j=1, \ldots, m$ we obtain

$$
\underline{X}=\sum_{j=1}^{m}\left(X_{j} e_{j}+Y_{j} \varepsilon_{j}\right)=\sum_{j=1}^{m}\left(X_{j}\left(\mathfrak{f}_{j}-\mathfrak{f}_{j}^{\dagger}\right)-Y_{j}\left(\mathfrak{f}_{j}+\mathfrak{f}_{j}^{\dagger}\right)\right)
$$

where $X_{\ell} \in \mathbb{R}, Y_{\ell} \in \mathbb{R}$, for $\ell=1, \ldots, m$. If we set $x_{j}:=X_{j}-Y_{j}, p_{j}:=X_{j}+Y_{j}$, we can write

$$
\sum_{j=1}^{m}\left(x_{j} \mathfrak{f}_{j}-p_{j} \mathfrak{f}_{j}^{\dagger}\right)=x-p^{\dagger}
$$


The variables $x, p$ are called affine vector variables. The vector derivative $\partial_{\underline{X}}$ in $\mathbb{R}_{m, m}$, which corresponds to a ultra-hyperbolic Dirac operator, is defined by

$$
\begin{aligned}
\partial_{\underline{X}} & =\sum_{j=1}^{2 m} e_{j} \partial_{X_{j}}=\sum_{j=1}^{m}\left(e_{j} \partial_{X_{j}}-\varepsilon_{j} \partial_{Y_{j}}\right) \\
& =2 \sum_{j=1}^{m}\left(\mathfrak{f}_{j} \partial_{p_{j}}-\mathfrak{f}_{j}^{\dagger} \partial_{x_{j}}\right)=2\left(\partial_{p^{\dagger}}-\partial_{x}\right) .
\end{aligned}
$$

Definition 3.1 The system

$$
\left\{\begin{array}{c}
\partial_{x} f\left(x, p^{\dagger}\right)=0 \\
\partial_{p^{\dagger}} f\left(x, p^{\dagger}\right)=0
\end{array}\right.
$$

is called affine monogenic system.

In this setting, it is possible to introduce a Fischer duality and a Fischer inner product which leads to a monogenic decomposition of homogeneous polynomials (see [20]).

Another generalization can be achieved by complexifying the vector variables and the Clifford algebra, obtaining the framework of the so-called Hermitian Clifford analysis, see $[2,3,16,19]$. Let us consider the complex Clifford algebra $\mathbb{C}_{m}$ endowed with the Witt basis. Let us introduce the variables

$$
\underline{z}=\sum_{j=1}^{m} \mathfrak{f}_{j} z_{j} \quad \underline{z}^{\dagger}=\sum_{j=1}^{m} \mathfrak{f}_{j}^{\dagger} z_{j}^{c},
$$

with $z_{j}=x_{j}+i x_{j+m}, j=1, \ldots, m$, and two complex Hermitian Dirac operators

$$
\partial_{\underline{z}}^{\dagger}=\sum_{j=1}^{m} \mathfrak{f}_{j} \partial_{z_{j}^{c}} \quad \partial_{\underline{z}}=\sum_{j=1}^{m} \mathfrak{f}_{j}^{\dagger} \partial_{z_{j}},
$$

where $\partial_{z_{j}^{c}}$ and $\partial_{z_{j}}$ denote the Cauchy-Riemann operator and its conjugate, in the variables $z_{j}$.

Definition 3.2 Let $f\left(\underline{z}, \underline{z}^{\dagger}\right)$ be a smooth function on an open set in $\mathbb{R}^{2 m}$ with values in the complex Clifford algebra $\mathbb{C}_{2 m}$. Then $f$ is called h-monogenic if it satisfies the system

$$
\left\{\begin{array}{c}
\partial_{z} f=0 \\
\partial_{\underline{z}}^{\dagger} f=0
\end{array}\right.
$$

Remark 3.3 For polynomial solutions of the Hermitian system the variables $z_{j}$ and $\bar{z}_{j}$ behave like fully independent and symbolic commuting variables. Even though the variables, in this case, are complex while in the affine case they are real, at a symbolic 
level the Hermitian and the affine system are similar. However the invariance of the two systems is different: the Hermitian system is invariant under the group $U(m)$ while the affine system is invariant under $\operatorname{GL}(m, \mathbb{R})$.

Remark 3.4 Subsequently, on the level of polynomials, the Hermitian and the affine monogenic systems are in fact fully equivalent. This is of course no longer true if one considers local solutions to the Hermitian (resp. affine) systems. In particular, the Hermitian system is elliptic while the affine system may assume distributional solutions.

A further generalization of Clifford Analysis is obtained in the next section in which we illustrate the basic of Metrodynamics in a specific case.

\subsection{Metrodynamics: the Symmetric Case}

In this section we recall some material from [5] to which we refer the reader for more details. Suppose we are given:

- a covariant rank 2 tensor:

$$
g=\left[g_{i j}\right], \quad g_{i j} \in \mathbb{R}, g_{i j}=g_{j i}, \quad 1 \leq i, j \leq m
$$

which is positive definite;

- the Grassmann algebra $\Lambda$ generated by the elements $\mathfrak{f}_{j}, j=1, \ldots, m$, satisfying the Grassmann relations

$$
\mathfrak{f}_{j} \mathfrak{f}_{k}+\mathfrak{f}_{k} \mathfrak{f}_{j}=0, \quad 1 \leq i, j \leq m
$$

- the dual Grassmann algebra $\Lambda^{\dagger}$ generated by $\mathfrak{f}^{\dagger}, j=1, \ldots, m$ satisfying the Grassmann relations

$$
\mathfrak{f}^{\dagger} \mathfrak{f}^{\dagger k}+\mathfrak{f}^{\dagger^{k}} \mathfrak{f}^{\dagger j}=0, \quad 1 \leq i, j \leq m
$$

The duality between the two Grassmann algebras is given by:

$$
\mathfrak{f}_{j} \mathfrak{f}^{\dagger}+\mathfrak{f}^{\dagger} \mathfrak{f}_{j}=\delta_{j}^{k} \text {. }
$$

Notation 3.5 In the sequel, we will adopt the Einstein summation convention, in other words, we will omit the symbol of summation when we are summing over equal contravariant and covariant indices. We will also make use of the following notation (due to Penrose): we set

$$
2 g_{(j k)}:=g_{j k}+g_{k j}, \quad 2 g_{[j k]}:=g_{j k}-g_{k j},
$$

and similarly for upper indices. 
Definition 3.6 Let $g=\left[g_{j k}\right], g_{j k} \in \mathbb{R}$, be a real covariant tensor of rank 2 and let us assume that it is positive definite and symmetric. We call $g$ the metric tensor.

Its reciprocal (contravariant) tensor is given by

$$
g^{j k}=\frac{1}{\operatorname{det}\left(g_{j k}\right)} G^{j k}
$$

where $G^{j k}$ denotes the cofactor of $g_{j k}$.

Note that

$$
g^{i j} g_{j k}=\delta_{k}^{i}
$$

where $\delta_{k}^{i}$ denotes the Kronecker's delta.

With the assumptions above, we can give the following:

Definition 3.7 The covariant basis for the Grassmann algebra $\Lambda^{\dagger}$ is given by the elements

$$
\mathfrak{f}_{j}^{\dagger}:=g_{j k} \mathfrak{f}^{\dagger}, j=1, \ldots, m \text {. }
$$

The contravariant basis for the Grassmann algebra $\Lambda$ is given by the elements

$$
\mathfrak{f}^{j}:=g^{j k} \mathfrak{f}_{k}, j=1, \ldots, m \text {. }
$$

We can introduce new covariant basis elements by

$$
e_{j}=\mathfrak{f}_{j}-g_{j k} \mathfrak{f}^{\dagger}, j=1, \ldots, m \text {. }
$$

It is easy to verify that they satisfy the relations $e_{j} e_{k}+e_{k} e_{j}=-2 g_{j k}$. An element $\left(x^{1}, \ldots, x^{m}\right) \in \mathbb{R}^{m}$ can be identified with the 1 -vector

$$
\underline{x}=x^{j} e_{j}=x^{j} \mathfrak{f}_{j}-x^{j} g_{j k} \mathfrak{\dagger}^{\dagger}
$$

thus identifying $\mathbb{R}^{m}$ with a subspace of the complex Clifford algebra $\mathbb{C}_{m}$. One may also consider the contravariant elements forming the so-called reciprocal basis:

$$
e^{j}=g^{j k} e_{k}, j=1, \ldots, m, e^{j} \in \operatorname{Span}\left\{e_{1}, \ldots, e_{m}\right\}
$$

Note that these elements can be obtained also as $e^{j}=\mathfrak{f}^{j}-\mathfrak{f}^{\dagger j}$. The following result is proved in [5]:

Proposition 3.8 For $j, k=1, \ldots, m$ we have the relations:

1. $e_{j} e^{k}+e^{k} e_{j}=-2 \delta_{j}^{k}$;

2. $\left\{e^{j}, e_{j}\right\}=-2 m$;

3. $\left[e^{j}, e_{j}\right]=0$. 
The notion of monogenicity is introduced through a Dirac operator which is the Fischer dual of the vector variable $\underline{x}$ and it is defined by

$$
\partial_{\underline{x}}=\partial_{x^{j}} e^{j}=\partial_{x^{j}} g^{j k} \mathfrak{f}_{k}-\partial_{x^{j}} \mathfrak{f}^{\dagger} .
$$

A smooth function $f\left(x^{1}, \ldots, x^{m}\right)=f(\underline{x})$ is called left monogenic on an open set $U$ in $\mathbb{R}^{m}$ if it satisfies $\partial_{\underline{x}} f(\underline{x})=0$ on $U$.

Recall that the Euler operator, which as it is well known measures the degree of homogeneity of polynomials, is defined by

$$
E_{\underline{x}}=x^{j} \partial_{x^{j}}
$$

The angular Dirac operator is defined by

$$
\Gamma_{\underline{x}}=-\frac{1}{2} x^{j} \partial_{x^{k}}\left(e_{j} e^{k}-e^{k} e_{j}\right)=-x^{j} \partial_{x^{k}} e_{j} \wedge e^{k}
$$

These two operators correspond to the scalar and the bivector part of the operator $\underline{x} \partial_{x}$, as stated in the first part of the following result (see [5]):

Proposition 3.9 The following relations hold:

1. $\underline{x} \partial_{\underline{x}}=\left[\underline{x} \partial_{\underline{x}}\right]_{0}+\left[\underline{x} \partial_{x}\right]_{2}=-E_{\underline{x}}-\Gamma_{\underline{x}}$;

2. $\underline{x} \underline{\partial_{x}}+\partial_{\underline{x}} \underline{x}=-m-2 E_{\underline{x}}$.

\subsection{Fischer Duality}

In the absolute setting, i.e. the orthogonal setting, it is possible to introduce the standard Fischer duality, which can be generalized to the covariant setting as follows. Let $\mathcal{P}$ be the algebra of polynomials generated by $\left\{x^{1}, \ldots, x^{m} ; e_{1}, \ldots, e_{m} ; i\right\}$, i.e. polynomials with coefficients in $\mathbb{C}_{m}$. The subset of homogeneous polynomials of degree $k$ will be denoted by $\mathcal{P}_{k}$ :

$$
\mathcal{P}_{k}=\left\{R_{k} \in \mathcal{P} \mid R_{k}(t \underline{x})=t^{k} R_{k}(\underline{x}), t \in \mathbb{R}\right\} .
$$

In $\mathcal{P}$ we can define the so-called Fischer inner product

$$
(R(\underline{x}), S(\underline{x}))=\left[\left\{R^{\dagger}\left(g^{1 j} \partial_{x^{j}}, \ldots, g^{m j} \partial_{x^{j}}\right)[S(\underline{x})]\right\}_{x=0}\right]_{0} .
$$

The differential operator $R^{\dagger}\left(g^{1 j} \partial_{x^{j}}, \ldots, g^{m j} \partial_{x^{j}}\right)$ has been obtained from $R(\underline{x})$ by taking its Hermitian conjugate followed by the substitution $x^{k} \rightarrow g^{k j} \partial_{x^{j}}$. The correspondences:

$$
\begin{aligned}
& e_{k} \stackrel{F}{\rightarrow}-e_{k} \\
& x^{k} \stackrel{F}{\rightarrow} g^{k j} \partial_{x^{j}}
\end{aligned}
$$


are called Fischer duality. Note that the first correspondence can be equivalently substituted by

$$
\mathfrak{f}_{k} \stackrel{F}{\rightarrow} \mathfrak{f}_{k}^{\dagger}=g_{k j} \mathfrak{f}^{\dagger j}
$$

We also have the so-called monogenic decomposition which is a consequence of the well known Fischer decomposition theorem (see [5]):

Theorem 3.10 (Monogenic decomposition) Any $R_{k} \in \mathcal{P}_{k}$ has a unique orthogonal decomposition of the form

$$
R_{k}(\underline{x})=\sum_{k=0}^{s} \underline{x}^{s} P_{k-s}(\underline{x}),
$$

where $\partial_{\underline{x}} P_{k-s}=0$.

\section{Metrodynamics: the General Case}

To justify the definitions we will give in the sequel, we start the section by discussing the underlying ideas. Suppose we are given the elements $\left\{\mathfrak{f}_{j}, j=1, \ldots, m\right\}$ generating a Grassmann algebra $\Lambda$ and $\left\{f^{\dagger}, j=1, \ldots, m\right\}$ generating a dual Grassmann algebra $\Lambda^{\dagger}$. Assume the duality relations $\mathfrak{f}_{j} \mathfrak{f}^{\dagger k}+\mathfrak{f}^{\dagger} \mathfrak{f}_{j}=\delta_{j}^{k}$. We start from the affine setting and we set (compare with (2)):

$$
\underline{X}=x^{j} \mathfrak{f}_{j}-p_{j} \mathfrak{f}^{\dagger j} .
$$

If we set $p_{j}=y^{k} g_{k j}$, where $g=\left[g_{j k}\right]$ is real, nonsingular, but not necessarily symmetric, we get that $x^{j}, y^{j}$ are now both contravariant variables in a vector space $\mathbb{R}^{m}$.

Let us put

$$
X^{j}=\frac{1}{2}\left(x^{j}+y^{j}\right) \quad Y^{j}=\frac{1}{2}\left(-x^{j}+y^{j}\right)
$$

so we can write the vector variable $\underline{X}$ in the form:

$$
\underline{X}=X^{j} e_{j}+Y^{j} \varepsilon_{j}
$$

where we have defined

$$
\mathfrak{f}_{j}^{\dagger}=g_{j k} \mathfrak{f}^{\dagger}, \quad e_{j}=\mathfrak{f}_{j}-\mathfrak{f}_{j}^{\dagger}, \quad \varepsilon_{j}=-\mathfrak{f}_{j}-\mathfrak{f}_{j}^{\dagger}, \quad j=1, \ldots, m .
$$

The elements $e_{j}, \varepsilon_{j}$ form the so-called $g$-basis. We have the following: 
Proposition 4.1 The elements $e_{j}, \varepsilon_{j}$ satisfy the relations:

$$
e_{j} e_{k}+e_{k} e_{j}=-2 g_{(j k)} ; \quad \varepsilon_{j} \varepsilon_{k}+\varepsilon_{k} \varepsilon_{j}=2 g_{(j k)} ; \quad e_{j} \varepsilon_{k}+\varepsilon_{k} e_{j}=2 g_{[j k]} .
$$

Proof

$$
\begin{aligned}
& e_{j} e_{k}+e_{k} e_{j}=\left(\mathfrak{f}_{j}-\mathfrak{f}_{j}^{\dagger}\right)\left(\mathfrak{f}_{k}-\mathfrak{f}_{k}^{\dagger}\right)+\left(\mathfrak{f}_{k}-\mathfrak{f}_{k}^{\dagger}\right)\left(\mathfrak{f}_{j}-\mathfrak{f}_{j}^{\dagger}\right) \\
& \quad=\left(\mathfrak{f}_{j} \mathfrak{f}_{k}+\mathfrak{f}_{k} \mathfrak{f}_{j}\right)+\left(\mathfrak{f}_{j}^{\dagger} \mathfrak{f}_{k}^{\dagger}+\mathfrak{f}_{k}^{\dagger} \mathfrak{f}_{j}^{\dagger}\right)-\left(\mathfrak{f}_{j} \mathfrak{f}_{k}^{\dagger}+\mathfrak{f}_{k}^{\dagger} \mathfrak{f}_{j}\right)-\left(\mathfrak{f}_{j}^{\dagger} \mathfrak{f}_{k}+\mathfrak{f}_{k} \mathfrak{f}_{j}^{\dagger}\right)=-g_{k j}-g_{j k},
\end{aligned}
$$

and in analogue way for the second relation. Finally:

$$
\begin{aligned}
e_{j} \varepsilon_{k}+\varepsilon_{k} e_{j} & =\left(\mathfrak{f}_{j}-\mathfrak{f}_{j}^{\dagger}\right)\left(-\mathfrak{f}_{k}-\mathfrak{f}_{k}^{\dagger}\right)+\left(-\mathfrak{f}_{k}-\mathfrak{f}_{k}^{\dagger}\right)\left(\mathfrak{f}_{j}-\mathfrak{f}_{j}^{\dagger}\right) \\
& =-\left(\mathfrak{f}_{j} \mathfrak{f}_{k}^{\dagger}+\mathfrak{f}_{k}^{\dagger} \mathfrak{f}_{j}\right)+\left(\mathfrak{f}_{j}^{\dagger} \mathfrak{f}_{k}+\mathfrak{f}_{k} \mathfrak{f}_{j}^{\dagger}\right)=-g_{k j}+g_{j k} .
\end{aligned}
$$

Remark 4.2 The options $y^{j}= \pm x^{j}$ give rise to the special vector variables $\underline{X}=X^{j} e_{j}$ and $\underline{X}=Y^{j} \varepsilon_{j}$.

The reciprocal basis of the $g$-basis can be obtained as follows. Suppose that we are given, as before, $\Lambda, \Lambda^{\dagger}$ and $g=\left[g_{j k}\right]$ a covariant tensor of rank 2 real and nonsingular. The reciprocal tensor of $g$ is given by $\left[g^{j k}\right]$ (see Definition 3.6). Consider the Dirac operator associated to the vector variable $\underline{X}$ :

$$
\partial_{\underline{X}}=2\left(\partial_{p_{j}} \mathfrak{f}_{j}-\partial_{x^{j}} \mathfrak{f}^{\dagger j}\right)
$$

Since we have defined $p_{j}=y^{k} g_{k j}$ and since the Euler operator $p_{j} \partial_{p_{j}}$ can be equivalently written as $y^{j} \partial_{y^{j}}$, we have $\partial_{p_{j}}=g^{j k} \partial_{y^{k}}$ and also

$$
\partial_{p_{j}} \mathfrak{f}_{j}=\partial_{y^{k}} g^{j k} \mathfrak{f}_{j}=\partial_{y^{k}} \mathfrak{f}^{k},
$$

where we have set

$$
\mathfrak{f}^{k}=\mathfrak{f}_{j} g^{j k} \text {. }
$$

We can use the elements $\mathfrak{f}_{j}^{\dagger}$ and the $g$-basis (5) to write the Dirac operator $\partial_{\underline{X}}$ as follows:

$$
\begin{aligned}
& \partial_{\underline{X}}=2\left(\partial_{p_{j}} \mathfrak{f}_{j}-\partial_{x^{j}} \mathfrak{f}^{\dagger j}\right)=2\left(\partial_{y^{k}} g^{j k} \mathfrak{f}_{j}-\partial_{x^{j}} \mathfrak{f}^{\dagger j}\right) \\
& \quad=2\left(\partial_{y^{j}} g^{k j} \mathfrak{f}_{k}-\partial_{x^{j}} g^{j k} \mathfrak{f}_{k}^{\dagger}\right)=\partial_{x^{j}} g^{j k}\left(e_{k}+\varepsilon_{k}\right)+\partial_{y^{j}} g^{k j}\left(e_{k}-\varepsilon_{k}\right)
\end{aligned}
$$

and

$$
\partial_{X^{j}}=\partial_{x^{j}}+\partial_{y^{j}} \quad \partial_{Y^{j}}=-\partial_{x^{j}}+\partial_{y^{j}}
$$


we does obtain

$$
\partial_{\underline{X}}=\frac{1}{2}\left(\partial_{X^{j}}-\partial_{Y^{j}}\right) g^{j k}\left(e_{k}+\varepsilon_{k}\right)+\frac{1}{2}\left(\partial_{X^{j}}+\partial_{Y^{j}}\right) g^{k j}\left(e_{k}-\varepsilon_{k}\right) .
$$

Again using the Penrose notation for upper indices, we can write the Dirac operator as

$$
\partial_{\underline{X}}=\partial_{X^{j}}\left(g^{(j k)} e_{k}+g^{[j k]} \varepsilon_{k}\right)+\partial_{Y^{j}}\left(-g^{(j k)} \varepsilon_{k}-g^{[j k]} e_{k}\right):=\partial_{X^{j}} e^{j}-\partial_{Y^{j}} \varepsilon^{j}
$$

where we have set

$$
e^{j}:=g^{(j k)} e_{k}+g^{[j k]} \varepsilon_{k} \quad \varepsilon^{j}:=g^{(j k)} \varepsilon_{k}+g^{[j k]} e_{k} .
$$

The elements $e^{j}, \varepsilon^{j}$ form the reciprocal $g$-basis.

Remark 4.3 The options $\partial_{y^{j}}= \pm \partial_{x^{j}}$ give rise to the special case of Dirac operators $\partial_{\underline{X}}=\partial_{X^{j}} e^{j}$ and $\partial_{\underline{X}}=\partial_{Y^{j}} \varepsilon^{j}$.

Remark 4.4 Note that $e^{j} \notin \operatorname{Span}\left\{e_{j} \mid j=1, \ldots, m\right\}$, unless $g^{[j k]}=0$ and $e^{j} \notin \operatorname{Span}\left\{\varepsilon_{j} \mid j=1, \ldots, m\right\}$, unless $g^{\{j k\}}=0$ thus, in general, we need to consider both the bases $e_{j}, \varepsilon_{j}$.

Proposition 4.5 We have the following reciprocal relations:

1. $e_{j} e^{k}+e^{k} e_{j}=-2 \delta_{j}^{k}$;

2. $\varepsilon_{j} \varepsilon^{k}+\varepsilon^{k} \varepsilon_{j}=2 \delta_{j}^{k}$;

3. $\left\{e_{j}, \varepsilon^{k}\right\}=\left\{\varepsilon_{j}, e^{k}\right\}=0$;

4. $\left\{e^{j}, e^{k}\right\}=-2 g^{(j k)},\left\{\varepsilon^{j}, \varepsilon^{k}\right\}=2 g^{(j k)},\left\{e^{j}, \varepsilon^{k}\right\}=2 g^{[j k]}$.

Proof Let us prove relation 1:

$$
\begin{aligned}
e_{j} e^{k}+e^{k} e_{j} & =e_{j}\left(g^{(k \ell)} e_{\ell}+g^{[k \ell]} \varepsilon_{\ell}\right)+\left(g^{(k \ell)} e_{\ell}+g^{[k \ell]} \varepsilon_{\ell}\right) e_{j} \\
& =g^{(k \ell)}\left(e_{j} e_{\ell}+e_{\ell} e_{j}\right)+g^{[k \ell]}\left(e_{j} \varepsilon_{\ell}+\varepsilon_{\ell} e_{j}\right) \\
& =-2 g^{(k \ell)} g_{(j \ell)}+2 g^{[k \ell]} g_{[j \ell]}=-2 \delta_{j}^{k} .
\end{aligned}
$$

Relation 2 can be proved in a similar way. Finally, we consider the first relation in 3 :

$$
\begin{aligned}
e_{j} \varepsilon^{k}+\varepsilon^{k} e_{j} & =e_{j}\left(g^{(k \ell)} \varepsilon_{\ell}+g^{[k \ell]} e_{\ell}\right)+\left(g^{(k \ell)} \varepsilon_{\ell}+g^{[k \ell]} e_{\ell}\right) e_{j} \\
& =g^{(k \ell)}\left(e_{j} \varepsilon_{\ell}+\varepsilon_{\ell} e_{j}\right)+g^{[k \ell]}\left(e_{j} e_{\ell}+e_{\ell} e_{j}\right) \\
& =2 g^{(k \ell)} g_{[j \ell]}-2 g^{[k \ell]} g_{(j \ell)}=0,
\end{aligned}
$$

the remaining relations can be proved in an analogous way. 
Example 4.6 Let us consider the simplest case, and let us take $m=1$. We introduce the metric $g=\left[g_{11}\right]$ with $g_{11} \neq 0$ and the Clifford algebra $\mathbb{R}_{1,1}$ generated by $e_{1}, \varepsilon_{1}$ and consider also the dual basis $\left\{e^{1}, \varepsilon^{1}\right\}$ which satisfies the relations $\left(e^{1}\right)^{2}=-\left(\varepsilon^{1}\right)^{2}=g^{11}$, $e^{1} \varepsilon^{1}+\varepsilon^{1} e^{1}=0$. The Dirac operator (10), in this case, is $\partial_{\underline{x}}=\partial_{x^{1}} e^{1}-\partial_{y^{1}} \varepsilon^{1}$. If we consider a function $f=f^{0}+f^{1} e^{1}+f^{2} \varepsilon^{1}+f^{3} e^{1} \varepsilon^{1}$ and we impose $\partial_{\underline{x}} f=0$ we get a system of four real equations which can be written in matrix form as

$$
\left[\begin{array}{cccc}
0 & -g^{11} x^{1} & -g^{11} y^{1} & 0 \\
x^{1} & 0 & 0 & g^{11} y^{1} \\
-y^{1} & 0 & 0 & -g^{11} x^{1} \\
0 & y^{1} & x^{1} & 0
\end{array}\right]\left[\begin{array}{l}
f^{0} \\
f^{1} \\
f^{2} \\
f^{3}
\end{array}\right]=0 .
$$

The matrix is of maximum rank since $g^{11} \neq 0$, and the characteristic variety turns out to be split into two linear subspaces of $\mathbb{R}^{2}: x^{1}= \pm y^{1}$.

We are now ready to introduce the general case of Metrodynamics in a more formal way. Let us introduce:

- a covariant rank 2 tensor:

$$
g=\left[g_{i j}\right], \quad g_{i j} \in \mathbb{R}, \quad \operatorname{det}(g) \neq 0,
$$

and

$$
g_{j \ell} g^{\ell k}=g^{k \ell} g_{\ell j}=\delta_{j}^{k}
$$

- the Grassmann algebra $\Lambda$ generated by the elements $\mathfrak{f}_{j}, j=1, \ldots, m$ satisfying the Grassmann relations;

- the dual Grassmann algebra $\Lambda^{\dagger}$ generated by $\mathfrak{f}^{\dagger}, j=1, \ldots, m$ satisfying the Grassmann relations;

the duality between $\Lambda$ and $\Lambda^{\dagger}$ is expressed by

$$
\mathfrak{f}_{j} \mathfrak{f}^{\dagger^{k}}+\mathfrak{f}^{\dagger^{k}} \mathfrak{f}_{j}=\delta_{j}^{k} \text {. }
$$

Remark 4.7 Note that the elements $\left\{\mathfrak{f}_{j}\right\}$ (resp. $\left.\left\{\mathfrak{f}^{\dagger j}\right\}\right)$ form a covariant tensor of rank 1.

We give the following:

Definition 4.8 The covariant basis for the Grassmann algebra $\Lambda^{\dagger}$ is given by the elements

$$
f_{j}^{\dagger}:=g_{j k} f^{\dagger}
$$

The contravariant basis for the Grassmann algebra $\Lambda$ is given by the elements

$$
\mathfrak{f}^{j}:=\mathfrak{f}_{k} g^{k j}
$$


Proposition 4.9 We have the following properties:

$$
\begin{aligned}
& \mathfrak{f}^{j} \mathfrak{f}^{k}+\mathfrak{f}^{k} \mathfrak{f}^{j}=0, \quad \mathfrak{f}^{j} \mathfrak{f}^{\dagger k}+\mathfrak{f}^{\dagger k} \mathfrak{f}^{j}=g^{k j} ; \\
& \mathfrak{f}_{j}^{\dagger} \mathfrak{f}_{k}^{\dagger}+\mathfrak{f}_{k}^{\dagger} \mathfrak{f}_{j}^{\dagger}=0, \quad \mathfrak{f}_{j} \mathfrak{f}_{k}^{\dagger}+\mathfrak{f}_{k}^{\dagger} \mathfrak{f}_{j}=g_{k j} ; \\
& \mathfrak{f}^{\dagger} \mathfrak{f}_{k}^{\dagger}+\mathfrak{f}_{k}^{\dagger} \mathfrak{f}^{\dagger j}=0, \quad \mathfrak{f}_{j}^{\dagger} \mathfrak{f}^{k}+\mathfrak{f}^{k} \mathfrak{f}_{j}^{\dagger}=\delta_{j}^{k} .
\end{aligned}
$$

Proof We have:

$$
\left\{\mathfrak{f}^{j}, \mathfrak{f}^{k}\right\}=\mathfrak{f}^{j} \mathfrak{f}^{k}+\mathfrak{f}^{k} \mathfrak{f}^{j}=\mathfrak{f}_{\ell} g^{\ell j} \mathfrak{f}_{i} g^{i k}+\mathfrak{f}_{i} g^{i k} \mathfrak{\mathfrak { f }}_{\ell} g^{\ell j}=\left(\mathfrak{f}_{\ell} \mathfrak{f}_{i}+\mathfrak{f}_{i} \mathfrak{f}_{\ell}\right) g^{\ell j} g^{i k}=0
$$

and similarly for the computation of $\left\{\mathfrak{f}_{j}^{\dagger}, \mathfrak{f}_{k}^{\dagger}\right\}$.

Moreover we have:

$$
\begin{gathered}
\mathfrak{f}_{j} \mathfrak{f}_{k}^{\dagger}+\mathfrak{f}_{k}^{\dagger} \mathfrak{f}_{j}=\mathfrak{f}_{j} g_{k \ell} \mathfrak{f}^{\dagger \ell}+g_{k \ell} \mathfrak{f}^{\dagger \ell} \mathfrak{f}_{j}=g_{k \ell}\left(\mathfrak{f}_{j} \mathfrak{f}^{\dagger^{\ell}}+\mathfrak{f}^{\dagger \ell} \mathfrak{f}_{j}\right)=g_{k \ell} \delta_{j}^{\ell}=g_{k j}, \\
\mathfrak{f}^{j} \mathfrak{f}^{\dagger k}+\mathfrak{f}^{\dagger^{k}} \mathfrak{f}^{j}=\mathfrak{f}_{\ell} g^{\ell j} \mathfrak{f}^{\dagger^{k}}+\mathfrak{f}^{\dagger k} \mathfrak{f}_{\ell} g^{\ell j}=g^{\ell j}\left(\mathfrak{f}_{\ell} \mathfrak{f}^{\dagger k}+\mathfrak{f}^{\dagger k} \mathfrak{f}_{\ell}\right)=g^{\ell j} \delta_{\ell}^{k}=g^{k j} .
\end{gathered}
$$

Finally

$$
\mathfrak{f}^{\dagger j} \mathfrak{f}_{k}^{\dagger}+\mathfrak{f}_{k}^{\dagger} \mathfrak{f}^{\dagger^{j}}=\mathfrak{f}^{\dagger j} g_{k \ell} \mathfrak{f}^{\dagger^{\ell}}+g_{k \ell} \mathfrak{f}^{\dagger^{\ell}} \mathfrak{f}^{\dagger j}=g_{k \ell}\left(\mathfrak{f}^{\dagger^{j}} \mathfrak{f}^{\mathfrak{f}^{\ell}}+\mathfrak{f}^{\dagger^{\ell}} \mathfrak{f}^{\dagger^{j}}\right)=0,
$$

and

$$
\mathfrak{f}_{j}^{\dagger} \mathfrak{f}^{k}+\mathfrak{f}^{k} \mathfrak{f}_{j}^{\dagger}=g_{j s} \mathfrak{f}^{\dagger s} \mathfrak{f}_{\ell} g^{\ell k}+\mathfrak{f}_{\ell} g^{\ell k} g_{j s} \mathfrak{f}^{\dagger s}=g_{j s} g^{\ell k}\left(\mathfrak{f}^{\dagger s} \mathfrak{f}_{\ell}+\mathfrak{f}_{\ell} \mathfrak{f}^{\dagger s}\right)=g_{j s} g^{\ell k} \delta_{\ell}^{s}=\delta_{j}^{k} .
$$

Remark 4.10 By simple computations, one obtains the following reciprocal relations:

$$
\mathfrak{f}_{k}=\mathfrak{f}^{j} g_{j k} \quad \mathfrak{f}^{\dagger k}=g^{k j} \mathfrak{f}_{j}^{\dagger} .
$$

Definition 4.11 Given the elements $\mathfrak{f}_{j}, \mathfrak{f}_{j}^{\dagger}$ we can define new covariant basis elements $e_{j}, e_{m+j}$ as follows:

$$
e_{j}:=\mathfrak{f}_{j}-\mathfrak{f}_{j}^{\dagger}, j=1, \ldots, m \quad e_{m+j}:=i\left(\mathfrak{f}_{j}+\mathfrak{f}_{j}^{\dagger}\right), j=1, \ldots, m .
$$

In the complex vector space

$$
\operatorname{Span}_{\mathbb{C}}\left\{\mathfrak{f}_{1}, \ldots, \mathfrak{f}_{m}\right\} \oplus \operatorname{Span}_{\mathbb{C}}\left\{\mathfrak{f}_{1}^{\dagger}, \ldots, \mathfrak{f}_{m}^{\dagger}\right\}
$$

one has the two complex subspaces $\operatorname{Span}_{\mathbb{C}}\left\{e_{1}, \ldots, e_{m}\right\}$ and $\operatorname{Span}_{\mathbb{C}}\left\{e_{m+1}, \ldots, e_{2 m}\right\}$ where the new basis elements satisfy the relations listed in the following Proposition. 
Proposition 4.12 For any $1 \leq j, k \leq m$ the following relations hold:

1. $e_{j} e_{k}+e_{k} e_{j}=-2 g_{(j k)}$;

2. $e_{m+j} e_{m+k}+e_{m+k} e_{m+j}=-2 g_{(j k)}$;

3. $e_{j} e_{m+k}+e_{m+k} e_{j}=-2 i g_{[j k]}$.

Proof We have:

$$
\begin{aligned}
e_{j} e_{k}+e_{k} e_{j} & =\left(\mathfrak{f}_{j}-\mathfrak{f}_{j}^{\dagger}\right)\left(\mathfrak{f}_{k}-\mathfrak{f}_{k}^{\dagger}\right)+\left(\mathfrak{f}_{k}-\mathfrak{f}_{k}^{\dagger}\right)\left(\mathfrak{f}_{j}-\mathfrak{f}_{j}^{\dagger}\right) \\
& =-\left(\mathfrak{f}_{j} \mathfrak{f}_{k}^{\dagger}+\mathfrak{f}_{k}^{\dagger} \mathfrak{f}_{j}\right)-\left(\mathfrak{f}_{j}^{\dagger} \mathfrak{f}_{k}+\mathfrak{f}_{k} \mathfrak{f}_{j}^{\dagger}\right)=-g_{j k}-g_{k j} . \\
e_{m+j} e_{m+k}+e_{m+k} e_{m+j} & =-\left(\mathfrak{f}_{j}+\mathfrak{f}_{j}^{\dagger}\right)\left(\mathfrak{f}_{k}+\mathfrak{f}_{k}^{\dagger}\right)-\left(\mathfrak{f}_{k}+\mathfrak{f}_{k}^{\dagger}\right)\left(\mathfrak{f}_{j}+\mathfrak{f}_{j}^{\dagger}\right) \\
& =-\left(\mathfrak{f}_{j} \mathfrak{f}_{k}^{\dagger}+\mathfrak{f}_{k}^{\dagger} \mathfrak{f}_{j}+\mathfrak{f}_{j}^{\dagger} \mathfrak{f}_{k}+\mathfrak{f}_{k} \mathfrak{f}_{j}^{\dagger}\right)=-g_{j k}-g_{k j},
\end{aligned}
$$

and similarly, we can compute

$$
e_{j} e_{m+k}+e_{m+k} e_{j}=i g_{j k}-i g_{k j}=-2 i g_{[j k]} \text {. }
$$

Remark 4.13 The basis elements $e_{m+j}, j=1, \ldots m$ are related to the elements $\varepsilon_{j}$ introduced above by

$$
\varepsilon_{j}=i e_{m+j}, \quad j=1, \ldots, m \text {. }
$$

Besides the covariant basis, we can introduce the contravariant basis:

Definition 4.14 Given the elements $\mathfrak{f}^{j}, \mathfrak{f}^{\dagger}$ we can define new contravariant basis elements $e^{i}, e^{m+i}$ defined by

$$
e^{j}:=\mathfrak{f}^{j}-\mathfrak{f}^{\dagger j}, \quad j=1, \ldots, m \quad e^{m+j}:=i\left(\mathfrak{f}^{j}+\mathfrak{f}^{\dagger j}\right), \quad j=1, \ldots, m .
$$

Remark 4.15 The complex Clifford algebra $\mathbb{C}_{m}[g]$ can be generated (among other possible choices) by the covariant basis $\left\{e_{j}, j=1, \ldots, m\right\}$ or the contravariant basis $\left\{e^{j}, j=1, \ldots, m\right\}$.

\section{$5 g$-basis Clifford Analysis}

\subsection{The General Case}

In this case, we can consider the choices $y^{j}=x^{j}, \partial_{y^{j}}=\partial_{x^{j}}$, and $y^{j}=-x^{j}$, $\partial_{y^{j}}=-\partial_{x^{j}}$.

Let us provide a motivation for the first choice. If we consider functions $f=f\left(X^{j}\right)$ i.e. functions which are constant with respect to the variables $Y^{j}$, then (10) becomes

$$
\partial_{\underline{X}}=\partial_{X^{j}}\left(g^{(j k)} e_{k}+g^{[j k]} \varepsilon_{k}\right) .
$$


However, $f\left(X^{j}\right)=f\left(\frac{1}{2}\left(x^{j}+y^{j}\right)\right)$ is determined by the restriction to the diagonal $x^{j}=y^{j}$, so we have (compare with (9))

$$
\begin{aligned}
\underline{X} & =\underline{x}=x^{j} e_{j} \\
\partial_{\underline{X}} & =\partial_{\underline{x}}=\partial_{x^{j}}\left(g^{(j k)} e_{k}+g^{[j k]} \varepsilon_{k}\right)=\partial_{x^{j}} e^{j} .
\end{aligned}
$$

Example 5.1 Let us consider again the simplest case i.e. let us take $m=1$. We introduce the metric $g=\left[g_{11}\right]$ with $g_{11} \neq 0$ and the Clifford algebra $\mathbb{R}_{1,1}$ generated by $e_{1}$, $\varepsilon_{1}$ (see Example 4.6). The Dirac operator reduces to $\partial_{x}=\partial_{x^{1}} g^{(11)} e_{1}=\partial_{x^{1}} e^{1}$. The matrix associated to the system, which is elliptic, can be formally obtained from (12) by setting $y^{1}=0$.

Example 5.2 Let us consider the case $m=2$ and the metric $g$ given by

$$
g=\left[\begin{array}{ll}
1 & \lambda \\
0 & 1
\end{array}\right]
$$

Consider the Clifford algebra $\mathbb{R}_{2,2}$ generated by $e_{1}, e_{2}, \varepsilon_{1}, \varepsilon_{2}$ and the dual basis $e^{1}, e^{2}$, $\varepsilon^{1}, \varepsilon^{2}$ satisfying the relations (see Proposition 4.5)

$$
\begin{aligned}
& \left(e^{1}\right)^{2}=\left(e^{2}\right)^{2}=-1, \quad\left(\varepsilon^{1}\right)^{2}=\left(\varepsilon^{2}\right)^{2}=1, \quad e^{i} \varepsilon^{i}+\varepsilon^{i} e^{i}=0, \\
& e^{1} e^{2}+e^{2} e^{1}=\lambda, \quad \varepsilon^{1} \varepsilon^{2}+\varepsilon^{2} \varepsilon^{1}=-\lambda, \quad e^{i} \varepsilon^{j}+\varepsilon^{j} e^{i}=(-1)^{i} \lambda, \quad i \neq j .
\end{aligned}
$$

A function $F$ with values in $\mathbb{R}_{2,2}$ has 16 real components and the condition $\partial_{\underline{x}} F=0$, with $\partial_{\underline{x}}=\partial_{x^{1}} e^{1}+\partial_{x^{2}} e^{2}$ gives rise to a system of 16 equations which can be written in matrix form as $A \vec{F}=\overrightarrow{0}$ where $\vec{F}$ denotes the vector made by the components of $F$. As it is well known (see for example [8]) from the matrix $A$ one can obtain information of the system. The characteristic variety is given, as a set, by $\lambda x^{1} x^{2}-\left(x^{1}\right)^{2}-\left(x^{2}\right)^{2}$ which is a quadric degenerate into a linear variety counted twice when $\lambda= \pm 2$. The cases $\lambda= \pm 2$ are special from another point of view: the so-called symmetric part of metric $g$, i.e. the metric $g^{\prime}$ defined by $g_{j k}^{\prime}=g_{(j k)}$ is degenerate. The resolution associated to the matrix $A$ ends in one step, as it happens in the case of the classical Dirac operator. The resolution in the case of several operators $\partial_{\underline{x}_{\ell}}, \ell=1, \ldots, n$ deserves a separate study. However, in the case of two or three operators one can directly verify, by using for example CoCoA [7], that for randomly chosen values of $\lambda$ the resolution is linear. In this sense, the system of several operators behaves like the standard Dirac system for functions with values in $\mathbb{R}_{2}$, see [8]. In analogous way, one can treat the case of the operator $\partial_{x^{1}} \varepsilon^{1}+\partial_{x^{2}} \varepsilon^{2}$, by associating a matrix $B$ to it. We obtain the same type of conclusions as in the case of the operator $\partial_{x^{1}} e^{1}+\partial_{x^{2}} e^{2}$, both in the case of one and several operators.

Remark 5.3 As the previous example shows, even when $g$ is invertible, there are several types of interesting cases depending on the symmetric part $g_{(j k)}$ and the antisymmetric part $g_{[j k]}$ of the metric $g$. 
From Proposition 4.5 we immediately get the following:

Proposition 5.4 The square of the Dirac operator $\partial_{\underline{x}}$ is given by

$$
\partial_{\underline{x}}^{2}=-\partial_{x^{k}} \partial_{x^{j}} g^{(j k)}:=-\Delta_{g, \underline{x}} .
$$

Definition 5.5 We call the operator

$$
\Delta_{g, \underline{x}}=\partial_{x^{k}} \partial_{x^{j}} g^{(j k)}
$$

$g$-Laplacian.

Remark 5.6 Note that for the Dirac operator (10) one has:

$$
\partial_{\underline{X}}^{2}=-\partial_{X^{k}} \partial_{X^{j}} g^{(j k)}+\partial_{Y^{k}} \partial_{Y^{j}} g^{(j k)}-\partial_{X^{k}} \partial_{Y^{j}} g^{[j k]} \text {. }
$$

Remark 5.7 1. In order to characterize the solutions $f$ of $\partial_{\underline{x}} f=g$ one may to solve the equation $\Delta_{g, x} h=g$ and put $f=-\partial_{\underline{x}} h$. Note that not all the solutions can be obtained in this way.

2. If $g$ is singular, the operator $\Delta_{g, x}$ is not surjective and $\partial_{\underline{x}}$ is not elliptic. This happens e.g. in the above Example 5.2 for $\lambda= \pm 2$.

The second choice: $y^{j}=-x^{j}, \partial_{y^{j}}=-\partial_{x^{j}}$ gives:

$$
-\underline{X}=\underline{x} \mid=x^{j} \varepsilon_{j}
$$

and

$$
-\partial_{\underline{X}}=\partial_{\underline{x}}=\partial_{x^{j}}\left(g^{(j k)} \varepsilon_{k}+g^{[j k]} e_{k}\right)
$$

The description is analogue to that one of the previous case.

Example 5.8 We consider again the case already treated in Example 5.2, i.e. the Clifford algebra $\mathbb{R}_{2,2}$ generated by $e_{1}, e_{2}, \varepsilon_{1}, \varepsilon_{2}$ and with dual basis $e^{1}, e^{2}, \varepsilon^{1}, \varepsilon^{2}$ satisfying the relations in Proposition 4.5. To compute the matrix associated to the general Dirac operator (10) we can make use of the matrices $A, B$ computed in Example 5.2. Indeed, the matrix of general Dirac operator corresponds to $A-B$. In the case of two and three operators, we can compute the resolution with the use of CoCoA. The resolutions are not anymore linear and have the same degree of the maps and same number of relations as the resolution for two or three Cauchy-Fueter operators respectively. This is not surprising, in fact the operator (10) can be written in the form (7) which is independent of the metric $g$ and depends only on the dimension of the algebra considered. Thus the resolutions in the case of several Dirac operators (10) are similar to the resolutions for the standard Dirac operator in the same dimension. 
Remark 5.9 The consideration of the choices $x_{i}=y_{i}$ or $x_{i}=-y_{i}$ is one of the possible options one can take. In general one can also consider the two operators $\partial_{\underline{x}}=\partial_{x^{j}} e^{j}$ and $\partial_{\underline{y} \mid}=\partial_{y^{j}} \varepsilon^{j}$ separately. The system of equations

$$
\partial_{\underline{x}} f=\partial_{\underline{y}} f=0
$$

is a generalization of the biregular system $\partial_{\underline{x}} f=f \partial_{y}=0$ which is standard in Clifford analysis (see [6,17], and [10] for more recent results on the biregular quaternionic case). Indeed with respect to the standard metric $g_{j k}=\delta_{j k}$ we have that

$$
\partial_{\underline{y} \mid} f I=\tilde{f} \partial_{\underline{y}} I
$$

so that $\partial_{\underline{x}} f I=\partial_{y \mid} f I$ for an $\mathbb{R}_{m}$-valued function $f$, reduces to $\partial_{\underline{x}} f=\tilde{f} \partial_{y}=0$, which is equivalent to the biregular system. More in general, one may consider systems involving several vector derivatives like $\partial_{\underline{x}}, \partial_{\underline{x}}, \partial_{\underline{y}}, \partial_{\underline{y}}$ etc., and in particular the system:

$$
\partial_{\underline{x}} f=\partial_{\underline{x} \mid} f-\partial_{\underline{y}} f=\partial_{\underline{y} \mid} f=0
$$

or the system:

$$
\partial_{\underline{x}} f=\partial_{\underline{y} \mid} f, \quad \partial_{\underline{x}} f=\partial_{\underline{y}} f .
$$

The strength of Clifford analysis lies in the huge class of special interesting systems which it provides.

\subsection{The Symplectic Case}

Let us suppose that $g_{j k}=-g_{k j}$, i.e. $g_{j k}=g_{[j k]}, g_{(j k)}=0$. This case can exist only if $m=2 n$, otherwise $g$ is singular.

Let us set, as in (5), $e_{j}=\mathfrak{f}_{j}-\mathfrak{f}_{j}^{\dagger}, \varepsilon_{j}=-\mathfrak{f}_{j}-\mathfrak{f}_{j}^{\dagger}, \mathfrak{f}_{j}^{\dagger}=g_{j k} \mathfrak{f}^{\dagger}, e^{j}=g^{j k} \varepsilon_{k}$. The relations satisfied by the elements in the $g$-basis and its reciprocal basis become as in the next result:

Proposition 5.10 We have the following relations:

1. $e_{j} e_{k}+e_{k} e_{j}=0$;

2. $\varepsilon_{j} \varepsilon_{k}+\varepsilon_{k} \varepsilon_{j}=0$;

3. $e_{j} \varepsilon_{k}+\varepsilon_{k} e_{j}=2 g_{j k}=-2 g_{k j}$;

4. $e^{j} \varepsilon^{k}+\varepsilon^{k} e^{j}=2 g^{j k}=-2 g^{j k}$;

5. $e^{j} e^{k}+e^{k} e^{j}=0$;

6. $e_{j} e^{k}+e^{k} e_{j}=-2 \delta_{j}^{k}$.

Proof The proof of $1 \div 6$ follows from Propositions 4.1 and 4.5.

Corollary 5.11 The elements $\left\{e^{j}, e_{j}\right\}$ form a Witt basis of $\mathbb{C}_{m, m}$. 
Remark 5.12 The calculus corresponding to the vector variable $\underline{x}=x^{j} e_{j}$, and the Dirac operator $\partial_{\underline{x}}=\partial_{x^{j}} e^{j}$ is an affine calculus with invariance group $\operatorname{GL}(m)$.

Define the vector variables

$$
\underline{x}=x^{j} e_{j} ; \quad \underline{x} \mid=x^{j} \varepsilon_{j}
$$

and the corresponding Dirac operators

$$
\partial_{\underline{x}}=\partial_{x^{j}} g^{j k} \varepsilon_{k} ; \quad \partial_{\underline{x} \mid}=\partial_{x^{j}} g^{k j} e_{k} .
$$

Definition 5.13 Let $U$ be an open set in $\mathbb{R}^{2 m}$ and let $f: U \subseteq \mathbb{R}^{2 m} \rightarrow \mathbb{C}_{2 m}$ be a differentiable function. We say that $f$ is $\operatorname{Sp}(n)$-monogenic if $\partial_{\underline{x}} f=\partial_{\underline{x}} f=0$.

Remark 5.14 In the symplectic setting, the square of the Dirac operator $\partial_{\underline{x}}$ vanishes.

Remark 5.15 The above definition would lead to a symplectic version of the Hodge system, in fact:

$$
\begin{aligned}
& \frac{1}{2}\left(\partial_{\underline{x}}+\partial_{\underline{x}}\right)=-\partial_{x^{j}} g^{j k} \mathfrak{f}_{k}=\partial_{x^{j}} \mathfrak{f}^{j} \approx d^{*} \\
& \frac{1}{2}\left(\partial_{\underline{x}}-\partial_{\underline{x}}\right)=-\partial_{x^{j}} g^{j k} g_{k \ell} \mathfrak{f}_{\ell}^{\dagger}=-\partial_{x^{j}} \mathfrak{f}^{\dagger j} \approx d .
\end{aligned}
$$

In the sequel we will work in $\mathbb{R}^{m}$, with $m=2 n$. The canonical symplectic Dirac system that comes out of the metrodynamics considerations is the one corresponding to the standard symplectic metric $g$ associated to the matrix

$$
\left[\begin{array}{cc}
0 & I \\
-I & 0
\end{array}\right]
$$

where $I$ is the $n \times n$ identity matrix.

The operators which make up the system may be written into the form:

$$
\begin{aligned}
& \partial_{x}=\sum_{j=1}^{n} \partial_{x_{j}} \mathfrak{f}_{j}+\partial_{x_{j+n}} \mathfrak{f}_{j+n} \\
& \partial_{\check{x}}=\sum_{j=1}^{n} \partial_{x_{j+n}} \mathfrak{f}_{j}^{\dagger}-\partial_{x_{j}} \mathfrak{f}_{j+n}^{\dagger} .
\end{aligned}
$$

where $\mathfrak{f}_{j}=\frac{1}{2}\left(e_{j}-i e_{j+m}\right)$ and $\mathfrak{f}_{j}^{\dagger}=\frac{1}{2}\left(-e_{j}+i e_{j+m}\right)$.

It is now possible to combine both equations in a quaternion manner and to this end, we will extend the Clifford algebra in order to have quaternionic coefficients instead of complex coefficients. We replace the imaginary unit $i$ by a quaternion $-I$ and introduce $J$ such that $I J=-J I$ and $I^{2}=J^{2}=-1$. Then, using the fact that 
$J \mathfrak{f}_{j}^{\dagger}=-\mathfrak{f}_{j} J$, we obtain

$$
\partial_{x}+J \partial_{\check{x}}=\sum_{j=1}^{n}\left(\mathfrak{f}_{j}+\mathfrak{f}_{j+n} J\right) \partial_{x_{j}}+\left(\mathfrak{f}_{j+n}-\mathfrak{f}_{j} J\right) \partial_{x_{j+n}}
$$

This operator can be rewritten as

$$
\sum_{j=1}^{n}\left(\mathfrak{f}_{j}+\mathfrak{f}_{j+n} J\right)\left(\partial_{x_{j}}-J \partial_{x_{j+n}}\right)
$$

where $\mathfrak{f}_{j}+\mathfrak{f}_{j+n} J=\left(e_{j}+I e_{j+2 n}+J e_{j+n}+K e_{j+3 n}\right)$. We have the following immediate fact:

Proposition 5.16 Let $F$ be a solution to the symplectic system. Then it is a nullsolution to the operator (22).

If one considers the complex symplectic group, it is possible to replace two sets of real partial derivatives $\partial_{x_{j}}, \partial_{x_{j+n}}$ by complex ones, for instance $\partial_{x_{j}}-I \partial_{x_{j+2 n}}$ and $\partial_{x_{j+n}}-I \partial_{x_{j+3 n}}$ so that the operator $\partial_{x_{j}}-J \partial_{x_{j+n}}$ is in fact being replaced by a Fueter type operator $\partial_{q_{j}}=\partial_{x_{j}}-I \partial_{x_{j+2 n}}-J \partial_{x_{j+n}}-K \partial_{x_{j+3 n}}$.

The operator obtained corresponds to the one studied in [14] and we are then back in our setting for quaternionic Clifford analysis

$$
\sum_{j=1}^{n}\left(\mathfrak{f}_{j}+\mathfrak{f}_{j+n} J\right) \partial_{q_{j}}
$$

which generalizes the symplectic system in the sense of Proposition 5.16.

The generalized biregular system $\partial_{\underline{x}} f=\partial_{y \mid} f=0$ introduced in (18) can be obviously be considered also in the symplectic case. However, the operator $\partial_{\underline{x}}$ by itself and also the pair of operators associated to the generalized biregular system $\partial_{\underline{x}}, \partial_{y \mid}$ are invariant under a bigger group than $\operatorname{Sp}(n), m=2 n$. Indeed, according to Corollary 5.11, the elements $e_{j}, e^{j}$ form a Witt basis with primitive idempotent $J=e_{1} e^{1} \ldots e_{m} e^{m}$ and the group leaving $J$ invariant is $\operatorname{GL}(m)$. In fact to obtain the symplectic invariance one has to consider the group leaving both idempotents $I$ and $J$ invariant that is transforms

$$
X \rightarrow S X \bar{S}, \quad X \in \mathbb{R}_{m, m}, S \in \operatorname{Spin}(m, m)
$$

leaving invariant the spaces

$$
\begin{aligned}
V & =\operatorname{Span}\left\{\mathfrak{f}_{j}, j=1, \ldots, m\right\}, \quad V^{\prime}=\operatorname{Span}\left\{\mathfrak{f}^{\dagger}, j=1, \ldots, m\right\}, \\
W & =\operatorname{Span}\left\{e_{j}, j=1, \ldots, m\right\}, \quad W \mid=\operatorname{Span}\left\{e^{j}, j=1, \ldots, m\right\}
\end{aligned}
$$


(or $\operatorname{Span}\left\{\varepsilon_{j}, j=1, \ldots, m\right\}$ and $\operatorname{Span}\left\{\varepsilon^{j}, j=1, \ldots, m\right\}$ ). Note hereby that the spaces $V$ and $V^{\prime}$ are determined by the primitive idempotent $I$ via the equations: $V=\left\{v \in \mathbb{R}^{2 m}: v I=0\right\}, V^{\prime}=\left\{v^{\prime} \in R^{2 m}: I v^{\prime}=0\right\}$ and in a similar way $W$ and $W \mid$ are determined by $W=\left\{w \in \mathbb{R}^{2 m}: w J=0\right\}, W \mid=\left\{w\left|\in R^{2 m}: J w\right|=0\right\}$. Conversely the selection of a dual pair $\left(V, V^{\prime}\right)$ determines a Witt basis leading to the construction of a primitive idempotent $I$ (not depending on the choice of this Witt basis) and similarly $J$ is determined by $W$ and $W \mid$. This concludes into the following (see also [21]):

Proposition 5.17 The symplectic group $S p(n)$ corresponds to the subgroup of $\operatorname{Spin}(m, m)$ leaving I and J invariant, i.e.

$$
\operatorname{Sp}(n)=\{S \in \operatorname{Spin}(m, m): S I \bar{S}=I \text { and } S J \bar{S}=J\} .
$$

We conclude that a truely $\mathrm{Sp}(n)$-invariant biregular system might consist of operators $\partial_{\underline{x}}, \partial_{\underline{x}}$ and $\partial_{y}$ (and/or $\left.\partial_{y \mid}\right)$ or for example system (19) or (20).

\section{The Covariant Versus the Hermitian Fischer Duality}

Let us now consider the Fischer duality in the setting of Metrodynamics. Let $\mathcal{P}$ be the algebra of polynomials generated by $\left\{x^{1}, \ldots, x^{m} ; \mathfrak{f}_{1}, \ldots, \mathfrak{f}_{m} ; \mathfrak{f}^{\dagger^{1}}, \ldots, \mathfrak{f}^{\dagger m}\right\}$, i.e. polynomials with coefficients in $\mathbb{C}_{m}$. The subset of homogeneous polynomials of degree $k$ will be denoted by $\mathcal{P}_{k}$ :

$$
\mathcal{P}_{k}=\left\{R_{k} \in \mathcal{P} \mid R_{k}(t \underline{x})=t^{k} R_{k}(\underline{x}), t \in \mathbb{R}\right\} .
$$

The map $\eta$ such that:

$$
\begin{gathered}
x^{k} \stackrel{\eta}{\rightarrow} \partial_{p_{j}}=g^{j k} \partial_{x^{k}} \\
\mathfrak{f}_{j}^{\dagger}=g_{j k} \mathfrak{f}^{\dagger k} \stackrel{\eta}{\rightarrow} \mathfrak{f}_{j} \\
\mathfrak{f}^{j}=\mathfrak{f}_{k} g^{k j} \stackrel{\eta}{\rightarrow} \mathfrak{f}^{\dagger}
\end{gathered}
$$

is called Fischer duality. We obtain:

$$
\begin{aligned}
& x^{j} \mathfrak{f}_{j} \rightarrow \partial_{x^{j}} \mathfrak{f}^{\dagger j} \\
& x^{j} \mathfrak{f}_{j}^{\dagger} \rightarrow \partial_{x^{k}} g^{j k} \mathfrak{f}_{j}=\partial_{p_{j}} \mathfrak{f}_{j}=\partial_{x^{j}} \mathfrak{f}^{j} .
\end{aligned}
$$

Moreover we have the following:

Lemma 6.1 The Fischer duality $\eta$ implies the following induced dualities:

$$
\begin{aligned}
& x^{j} e_{j} \rightarrow-\partial_{x^{j}} e^{j} \\
& x^{j} \varepsilon_{j} \rightarrow \partial_{x^{j}} \varepsilon^{j} .
\end{aligned}
$$


Proof The dualities easily follow from the fact that $e_{j}=\mathfrak{f}_{j}-\mathfrak{f}_{j}^{\dagger}, \varepsilon_{j}=-\mathfrak{f}_{j}-\mathfrak{f}_{j}^{\dagger}$, the definition of $e^{j}, \varepsilon^{j}$ and the duality $F$ defined in Sect. 3.3.

In $\mathcal{P}$ we can define the so-called Fischer bilinear form

$$
(R(\underline{x}), S(\underline{x}))=\left[\{\eta(R(\underline{x}))[S(\underline{x})]\}_{\underline{x}=0}\right]_{0} .
$$

The Fischer bilinear form is nondegenerate.

Proposition 6.2 For any $R \in \mathcal{P}$ we have $(R(\underline{x}), \underline{x} A)=0$ if and only if $\partial_{\underline{x}} R(\underline{x})=0$.

Proof We have

$$
\begin{aligned}
0 & =(\underline{x} A, R(\underline{x}))=\left[\{\eta(\underline{x} A) R(\underline{x})\}_{\underline{x}=0}\right]_{0} \\
& =\left[\left\{\eta(A) \partial_{\underline{x}} R(\underline{x})\right\}_{\underline{x}=0}\right]_{0}=\left(A, \partial_{\underline{x}} R(\underline{x})\right)
\end{aligned}
$$

from which we deduce that $\partial_{\underline{x}} R(\underline{x})=0$ by the arbitrarity of $A$.

In a similar way one can prove the following:

Corollary 6.3 Let $R(\underline{x}), A, B \in \mathcal{P}$. We have

$$
(R(\underline{x}), \underline{x} A+\underline{x} \mid B)=0
$$

if and only if $R$ is $S p(n)-$ monogenic.

Proposition 6.4 In the symplectic case, the Fischer bilinear form gives:

$$
\left(e_{j}, e_{k}\right)=g_{k j} .
$$

Proof We have

$$
\eta\left(e_{j}\right)=-e^{k} g_{k j} \quad \eta\left(\varepsilon_{j}\right)=-\varepsilon^{k} g_{k j},
$$

thus

$$
\left(e_{j}, e_{k}\right)=-\left[e^{\ell} g_{\ell j} e_{k}\right]_{0}=g_{k j} \text {. }
$$

Theorem 6.5 (Fischer decomposition) Any $R \in \mathcal{P}$ has a unique decomposition of the form

$$
R(\underline{x})=P(\underline{x})+\underline{x} P_{1}(\underline{x})+\underline{x} \mid P_{2}(\underline{x}),
$$

where $P$ is $\operatorname{Sp}(n)-$ monogenic. 
We can introduce another Fischer duality which is related to that one of the Hermitian setting but will be more general, since it involves coordinates and metrics as well. Let us denote by $\xi$ the Hermitian Fischer duality defined by

$$
\begin{aligned}
& x^{j} \stackrel{\xi}{\stackrel{\xi}{\rightleftarrows}} \partial_{x^{j}} \\
& \mathfrak{f}_{j} \underset{\xi}{\stackrel{\xi}{\rightleftarrows}} \mathfrak{f}^{\dagger} \\
& g_{j k} \stackrel{\xi}{\rightleftarrows} \partial_{g_{j k}} .
\end{aligned}
$$

This duality $\xi$ does not respect covariance (compare with $\eta$ ) and depends on the choice of the absolute frame. However we have:

$$
\begin{gathered}
x=x^{j} \mathfrak{f}_{j} \stackrel{\stackrel{\xi}{\rightrightarrows}}{\longrightarrow} \partial_{x^{j}} \mathfrak{\dagger}^{j}=\partial_{x} \\
p^{\dagger}=p_{j} \mathfrak{f}^{\dagger} \stackrel{\xi}{\rightleftarrows} \partial_{p_{j}} \mathfrak{f}_{j}=\partial_{p^{\dagger}}
\end{gathered}
$$

thus $\xi$ preserves affine invariants.

If $g_{j k}$ are constant then one would think of defining

$$
p_{j}=y^{k} g_{k j} \stackrel{\xi}{\rightleftarrows} \partial_{p_{j}}=\partial_{y^{k}} g^{j k} .
$$

If the $g_{j k}$ are variable, then the Hermitian Fischer duality gives $g_{j k} \stackrel{\xi}{\rightleftarrows} \partial_{g_{j k}}$, so that we obtain the correspondence

$$
p_{j}=y^{k} g_{k j} \stackrel{\xi}{\rightleftarrows} \partial_{p_{j}}=\partial_{y^{k}} \partial_{g_{k j}} .
$$

Hence if we require $p_{j} \stackrel{\xi}{\rightleftarrows} \partial_{p_{j}}$, where the $p_{j}$ are variables independent of $y^{k}$ and $g_{j k}$ then we get the system of equations

$$
\left(\partial_{p_{j}}-\partial_{y_{k}} \partial_{g_{k j}}\right) f=0 .
$$

Conclusion. We conclude that the Metrodynamics as introduced in Sect. 4 is, in a sense, "static" as the $g_{j k}$ are fixed. One may obtain a completely new notion while considering $g_{j k}$ as variables. In this case, we will not require any assumption on the invertibility of $g$. In this new setting it will be possible to have a Fischer duality leading to a Fischer decomposition. The new Hermitian Fischer duality $\xi$ will allow in particular to associate to the variables $x$ and $p^{\dagger}$ the following operators:

$$
x=x^{j} \mathfrak{f}_{j} \stackrel{\xi}{\rightarrow} \partial_{x^{j}} \mathfrak{f}^{\dagger j}=\partial_{x} \quad p^{\dagger}=x^{j} g_{j k} \mathfrak{\dagger}^{\dagger} \stackrel{\xi}{\rightarrow} \partial_{x^{j}} \partial_{g_{j k}} \mathfrak{f}_{k}=\partial_{p^{\dagger}} .
$$

These operators lead to the following monogenic Hodge-Dirac-like system:

$$
\left\{\begin{array}{l}
\partial_{x} f(x, g)=0 \\
\partial_{p^{\dagger}} f(x, g)=0 .
\end{array}\right.
$$




\section{The Matrix Derivative}

To illustrate the several possibilities which can be treated in this new framework, we conclude the paper by introducing the metrodynamics point of view of the matrix derivative. Suppose that we are given, as in Sect. 4 , the elements $\mathfrak{f}_{j}, \mathfrak{f}^{\dagger j}, j=1, \ldots, m$ generating a Grassmann algebra $\Lambda$ and a dual Grassmann algebra $\Lambda^{\dagger}$, respectively, and a nondegenerate metric $g=\left[g_{i j}\right]$. Let $A=\left[A_{i j}\right]$ be a covariant matrix, i.e. a matrix whose entries are covariant elements. Let us consider

$$
\underline{A}=A_{i j} \mathfrak{f}^{i} \mathfrak{f}^{j}
$$

where, as in Sect. 4, we have set $\mathfrak{f}^{i}=\mathfrak{f}_{k} g^{k i}$. We can define the matrix derivatives:

$$
\partial_{\underline{A}}:=\mathfrak{f}_{j} \mathfrak{f}_{i}^{\dagger} \partial_{A_{i j}} .
$$

If we consider an Hermitian setting, we can replace the elements $\mathfrak{f}^{i}$ by $\mathfrak{f}_{k} \partial_{g_{i k}}$ (note that this replacement corresponds to take the Fischer dual of $\mathfrak{f}_{i}^{\dagger}=g_{i k} \mathfrak{f}^{\dagger}$ ). Thus we can set $\mathfrak{f}^{i}=\mathfrak{f}_{k} \partial_{g_{i k}}$ in $\underline{A}$ to obtain $\underline{A}=A_{i j} \mathfrak{f}_{k} \partial_{g_{i k}} f^{\dagger}$ and $\partial_{\underline{A}}$ can be seen as the Fischer dual of the variable $\underline{A}$, indeed we have:

$$
\partial_{\underline{A}}=\mathfrak{f}_{j} g_{i k} \mathfrak{f}^{\dagger k} \partial_{A_{i j}}=\mathfrak{f}_{j} \mathfrak{f}_{i}^{\dagger} \partial_{A_{i j}} .
$$

In the specific case in which $A_{i j}=g_{i j}$ we obtain:

$$
\underline{g}=g_{i j} \mathfrak{f}^{i} \mathfrak{f}^{j}=\mathfrak{f}_{j} \mathfrak{\dagger}^{\dagger} \in \operatorname{End}\left(\mathbb{R}_{m}\right),
$$

thus in the given basis, $g$ is a specific element in $\operatorname{End}\left(\mathbb{R}_{m}\right)$ independent of the component of $g$.

Moreover we have:

Proposition 7.1 We have the following

$$
\left[\underline{A}, \mathfrak{f}_{i}^{\dagger}\right]=-A_{i k} f^{\dagger^{k}}
$$

and

$$
A_{i j}=\left\{-\left[\underline{A}, \mathfrak{f}_{i}^{\dagger}\right], \mathfrak{f}_{j}\right\}
$$

Proof Simple computations using the relations in Proposition 4.9 show that

$$
\left[\underline{A}, \mathfrak{f}_{i}^{\dagger}\right]=A_{\ell k} \mathfrak{f}^{\ell} \mathfrak{f}^{\dagger} \mathfrak{f}_{i}^{\dagger}-\mathfrak{f}_{i}^{\dagger} A_{\ell k} \mathfrak{f}^{\ell} \mathfrak{f}^{\dagger k}=-A_{\ell k}\left\{\mathfrak{f}^{\ell}, \mathfrak{f}_{i}^{\dagger}\right\} \mathfrak{f}^{\dagger k}=-A_{i k} \mathfrak{f}^{\dagger k} ;
$$


and

$$
\left\{-\left[\underline{A}, \mathfrak{f}_{i}^{\dagger}\right], \mathfrak{f}_{j}\right\}=\left\{A_{i k} \mathfrak{f}^{\dagger}, \mathfrak{f}_{j}\right\}=A_{i k} \delta_{j}^{k}=A_{i j} .
$$

From the previous proposition we get, in particular, that

$$
g_{i j}=\left\{-\left[\underline{g}, \hat{f}_{i}^{\dagger}\right], \mathfrak{f}_{j}\right\}
$$

In the basis $\mathfrak{f}^{j}, \mathfrak{f}^{\dagger}$ we have that $g$ is now depending on $g$. We can introduce the operator

$$
\partial_{\underline{g}}=\mathfrak{f}_{i} \mathfrak{f}_{j}^{\dagger} \partial_{g_{i j}}
$$

leading to $\partial_{\underline{g}} f(\underline{x}, g)=0$ which translates into the so-called metrogenic system

$$
\partial_{\underline{x}} f(\underline{x}, g)=\partial_{g} f(\underline{x}, g)=0 .
$$

Proposition 7.2 If the metric $g$ is symmetric then

$$
\partial_{\underline{g}}=\underline{g} .
$$

Proof If we express $\underline{g}$ in the given basis, by replacing $\mathfrak{f}^{i}$ by $\mathfrak{f}_{k} \partial_{g_{i k}}$, we get

$$
\underline{g}=g_{i j} \partial_{g_{i k}} \mathfrak{f}_{k} \mathfrak{f}^{\dagger}
$$

and $\partial_{g}=\mathfrak{f}_{k} \mathfrak{f}_{i}^{\dagger} \partial_{k i}$ can be written as $\partial_{g}=g_{i j} \partial_{g_{k i}} \mathfrak{f}_{k} \mathfrak{f}^{\dagger}$. By the hypothesis on $g$ we get:

$$
\partial_{\underline{g}}=g_{i j} \partial_{g_{i k}} \mathfrak{f}_{k} \mathfrak{f}^{\dagger}=\underline{g} .
$$

To conclude notice that:

$$
\partial_{\underline{g}}+\overline{\partial_{g}}=g_{i j} \partial_{g_{i j}}=E_{g}
$$

where $E_{g}$ denotes the Euler operator.

Remark 7.3 One may introduce matrix variables and derivatives also with respect to the g-basis by $A=A_{j k} e^{i} e^{j+m}$ and $\partial_{A}=\partial_{A_{i j}} e_{i} e_{j+m}$. These operators behave in a very different way from the ones introduced above. It is clear that the theory of matrix operators and derivatives is a topic in its own which is beyond the scope of this paper. 


\section{References}

1. Brackx, F., Delanghe, R., Sommen, F.: Clifford Analysis. Research Notes in Mathematics, vol. 76, Pitman, London (1982)

2. Brackx, F., Bureš, J., De Schepper, H., Eelbode, D., Sommen, F., Souček, V.: Fundaments of Hermitian Clifford analysis-Part I : complex structure. Complex Anal. Oper. Theory. 1(3), 341-365 (2007)

3. Brackx, F., Bureš, J., De Schepper, H., Eelbode, D., Sommen, F., Souček, V.: Fundaments of Hermitian Clifford analysis-part II : splitting of $h$-monogenic equations. Fundaments of Hermitean Clifford analysis. II. Splitting of $h$-monogenic equations. Complex Var. Elliptic Equ. 52(10-11), 1063-1079 (2007)

4. Brackx, F., De Schepper, H., Sommen, F.: A Hermitian setting for wavelet analysis: the basics. In: Proceedings of the 4th International Conference on Wavelet Analysis and its Applications. University of Macau, China (2005)

5. Brackx, F., De Schepper, N., Sommen, F.: Metric dependent Clifford analysis with applications to wavelets analysis. In: Alpay, D. (ed.) Wavelets, multiscale systems and hypercomplex analysis, pp. 17-67, Oper. Theory Adv. Appl. 167, Birkhäuser, Basel (2006)

6. Brackx, F., Pincket, W.: A Bochner Martinelli formula for the biregular functions of Clifford analysis. Complex Var. Theor. Appl. 4, 39-48 (1984)

7. CoCoA Team, CoCoA: a system for doing Computations in Commutative Algebra, Available at http://cocoa.dima.unige.it

8. Colombo, F., Sabadini, I., Sommen, F., Struppa, D.C.: Analysis of Dirac Systems and Computational Algebra: Progress in Mathematical Physics, vol. 39, Birkhäuser, Boston (2004)

9. Crumeyrolle, A.: Orthogonal and Symplectic Clifford Algebras. Kluwer, Dordrecht (1990)

10. Damiano, A., Sabadini, I., Struppa, D.C.: New Algebraic Properties of Biregular Functions in $2 n$ Quaternionic Variables. Complex Var. Elliptic Equ. 51, 497-510 (2006)

11. Delanghe, R., Sommen, F., Souček, V.: Clifford Algebra and Spinor-valued Functions. Mathematics and its Applications 53, Kluwer, Dordrecht (1992)

12. Gilbert, J., Murray, M.A.M.: Clifford Algebras and Dirac Operators in Harmonic Analysis. Cambridge University Press, Cambridge (1991)

13. Lounesto, P.: Clifford Algebras and Spinors. London, Math. Soc. Lecture Notes Series, vol 286, Cambridge University Press, Cambridge (2001)

14. Peña-Peña, D., Sabadini, I., Sommen, F.: Quaternionic Clifford analysis: The Hermitian Setting. Complex Var. Oper. Theor. 1(1), 97-113 (2007)

15. Porteous, I.R.: Clifford Algebras and the Classical Groups. Cambridge Studies in Advanced Mathematics, vol. 50, Cambridge University Press, Cambridge (1995)

16. Rocha-Chavez, R., Shapiro, M., Sommen, F.: Integral theorems for functions and differential forms in $\mathbb{C}^{m}$. Research Notes in Math. 428, Chapman and Hall, CRC Press, Boca Raton, London (2001)

17. Sabadini, I., Sommen, F., Struppa, D.C.: Series and integral representations for the biregular exponential function. J. Nat. Geom. 21, 1-16 (2002)

18. Sabadini, I., Sommen, F., Struppa, D.C., Van Lancker, P.: Complexes of Dirac operators in Clifford algebras. Math. Z. 239, 293-320 (2002)

19. Sabadini, I., Sommen, F.: Hermitian Clifford analysis and resolutions. Clifford Anal. Appl. Math. Meth. Appl. Sci. 25(16-18), 1395-1413 (2002)

20. Sommen, F.: Defining a q-deformed version of Clifford analysis. Complex Variables Theory Appl. 34, 247-265 (1997)

21. Sommen, F., Van Acker, N.: $S O(m)$-invariant differential operators on Clifford algebra valued functions. Found. Phys. 23(11), 1491-1519 (1993) 